\title{
Compte rendu du symposium international ARES SERONO : \\ Male factor in human infertility
}

\author{
Neuilly, 21-22 Avril 1994
}

Ce symposium était divisé en 3 parties: données fondamentales, tests d'évaluation fonctionnelle et thérapeutique.

\section{Premier thème : Données fondamen- tales concernant les mécanismes de fécondation}

YANAGIMACHI (Honolulu) a fait un rappel des mécanismes de la fécondation chez les mammiferes. L'espèce humaine se distingue par le fait que le transit épididymaire est dans l'ensemble plus rapide, mais également très variable chez le même individu; au sein d'un éjaculat co-existeront des spermatozoïdes qui auront parcouru l'épididyme en 1 jour et d'autres en 12 jours: leur état de maturation respectif sera ainsi différent, ce qui peut expliquer les grandes variations concernant le temps de capacitation, entre les spermatozoïdes d'un même éjaculat.

Chez tous les mammifêres, le blocage de la polyspermie a lieu à 2 niveaux: zone pellucide (ZP) et ovolemme. Chez l'homme, il semble principalement limité à la région interne de la ZP. Chez la souris, le noyau de spermatides rondes est capable de participer à la syngamie: par électro-fusion, on a pu obtenir des naissances (avec un faible rendement néanmoins).

LYNE FRASER (Londres) a décrit l'environnement ionique favorable à la capacitation et à la réaction acrosomale (RA). Le $\mathrm{Ca}++$ joue un rôle clé dans les 2 processus, mais la régulation des concentrations de calcium intra cellulaire procéderait de 2 méca- nismes différents: mise en route d'une ATPase-calcium dépendante pendant la capacitation, ouverture de canaux calciques pour déclencher la RA. Le sodium $(\mathrm{Na}+)$ est également nécessaire, à doses plus fortes pour la RA que pour la capacitation. Pour déclencher la $\mathrm{RA}$, un échange $\mathrm{Na}+\mathrm{H}+$ élèverait le $\mathrm{pH}$ intracellulaire, entrainant l'activation de canaux calciques. Le potassium $(\mathrm{K}+)$, qui est 5 fois plus concentré dans les sécrétions tubaires que dans le sang, n'est pas nécessaire à la capacitation, et inhibe à fortes doses la RA.

Certains facteurs stimulent la capacitation: progestérone, pentoxifylline, et un peptide nouvellement découvert, proche du TRH. Toutes ces observations ont été réalisées en utilisant un marquage à la chlortetracycline fluorescente, permettant seule de révéler des spermatozoïdes capacités.

KRUGER (Afrique du Sud) a souligné l'importance des critères morphologiques, bons prédicteurs de la capacité fécondante d'un sperme (corrélation négative entre la tératozoospermie et le hamster test ainsi que la qualité du DNA (appréciée par la coloration à l'acridine orange).

AITKEN (Edimbourg) a montré l'intérêt de l'analyse automatisée du mouvement spermatique, tout en soulignant l'importance de conditions de mesures rigoureuses. Ainsi, l'hyperactivation est très sensible aux microvariations de température $\left(<1^{\circ} \mathrm{C}\right)$, qui peuvent être engendrées par la régulation 
électronique d'une platine chauffante. Aitken a également décrit les effets délétères dus aux radicaux libres dérivés de l'oxygène, sur la membrane spermatique. Celle-ci est riche en acides gras insaturés, et par là très sensible aux radicaux libres. Il existe des facteurs intrinsèques (activation d'un système "xanthine-oxydase", en relation avec une certaine immaturité cellulaire) et extrinsèques, comme la présence de leucocytes. L'albumine bovine, contenue dans des milieux du type "B2", ou le sulfate de fer contenu dans le Ham's, constituent également des sources de radicaux libres.

\section{Deuxième thème : Tests d'évaluation fonctionnelle des spermatozoïdes}

Cummins (Australie) a montré que la mesure du taux de réaction acrosomique spontanée a peu de valeur prédictive; la mesure après stimulation par un ionophore ou d'autres stimulants est plus intéressante. On peut utiliser des agents pharmacologiques comme la pentoxifylline, ou physiologiques, comme la progestérone, le fluide folliculaire, le cumulus, la ZP solubilisée, etc ...

Matson (Manchester) évoque l'utilisation des ovocytes humains non fécondés provenant de la fécondation in vitro, soit à l'état frais, soit après congélation et conservation dans l'azote liquide. Le problème est que la ZP est durcie au bout de 48 heures de culture, et qu'on peut donc avoir des faux-négatifs, si on veut tester la capacité de certains spermatozoïdes à pénétrer cette $\mathrm{ZP}$. On peut aussi éliminer la ZP par digestion enzymatique afin de réaliser un test de pénétration homo-spécifique. Ce test s'avère particulièrement intéressant si on se propose de réaliser ultérieurement une microinjection sous la ZP ("SUZI"), pour un sperme donné: si le test est négatif, il faudrait alors réaliser une injection intra-cytoplasmique ("ICSI") plutôt qu'une "SUZI".

LIU et GoRDON BAKER (Melbourne) ont fait une analyse de régression logistique afin de déterminer, parmi différents facteurs incluant les paramètres classiques du spermogramme, l'aptitude des spermatozoïdes à faire la RA, le test hyposmotique, le test au bleu d'aniline, etc.., lesquels étaient les mieux corrélés aux résultats de la fécondation in vitro (FIV). Les 2 facteurs les plus significatifs étaient le nombre de spermatozoïdes liés à la ZP, et la proportion de ZP pénétrées.

2 tests sont disponibles qui éliminent un effet "ovocyte" éventuel: (i) le test de l'hémizona, consiste à sectionner une ZP en 2 hémisphères, qui seront incubées en présence l'une de spermatozoïdes dont on veut tester la capacité de fixation, l'autre de spermatozoïdes témoins. (ii) un autre test consiste à incuber chaque $\mathrm{ZP}$ en présence de 2 lots de spermatozoïdes, l'un à tester, l'autre témoin, marqués chacun d'un fluorochrome différent (par exemple: FITC vert, et rhodamine rouge).

MENDOZA (Grenade) et TESARIK (Neuilly) ont clos ce chapitre "diagnostic" en décrivant des substituts synthétiques de la ZP humaine, qui reconnaissent les mêmes structures membranaires du spermatozoïde que les molécules de ZP (type ZP3) responsables de la liaison ZP-spermatozoïdes. Les meilleurs candidats actuels sont des glycoprotéines et mannosylés de la BSA. Il est intéressant de noter que si on connait relativement bien les molécules de la ZP impliquées dans la fixation, celles du spermatozoïde n'ont pas encore été clairement identifiées.

\section{Troisième thème : Les traitements dis- ponibles dans l'infertilité masculine}

Acosta (communication présentée par Kruger) a passé en revue les différents traitements médicamenteux qu'on peut proposer dans l'oligoasthénotératospermie dite "idiopathique":

(i) hormonaux: GnRH, gonadotropines, antiestrogènes, androgènes, inhibiteurs de l'aromatase, (ii) non hormonaux : kallikréine

Aucun traitement n'a fait les preuves de son efficacité contre un placebo (dans beaucoup 
d'études ce dernier est généralement plus efficace, à la limite de la significativité statistique!).

La FSH purifiée à haute doses (150 UI par jour tous les 2 jours) a permis d'augmenter "spectaculairement" les taux de fécondation en FIV (étude publiée il y a maintenant 3 ans, en 1991); mais on attend toujours une étude prospective randomisée !

Clara Mills (Londres) a montré les effets intéressants de la pentoxifylline si celle-ci est utilisée à bon escient, c'est à dire dans le but de corriger une déficience fonctionnelle des spermatozoïdes d'un patient, et non ajoutée systématiquement, à priori, dans le milieu de culture. Elle agit comme un inhibiteur de la phosphodiestérase en élevant la teneur en AMP cyclique intracellulaire, mais aurait d'autres effets, comme celui de protéger la membrane des radicaux libres. Elle augmente la vitesse curvilinéaire des spermatozoïdes, mais surtout l'hyperactivation, qui est un reflet de la capacitation. Elle est donc particulièrement indiquée dans les spermes qui présentent un défaut d'hyperactivation quand ils sont placés dans des conditions capacitantes.

CHECK (New Jersey, USA) a insisté sur la nécessité de trouver des critères rigoureux pour identifier un facteur masculin dans une stérilité de couple. Il a retenu le paramètre "concentration de mobiles" comme le plus discriminant, la valeur seuil étant située à 10 millions $/ \mathrm{ml}$, tandis que d'autres avaient peu de valeur, comme le test hypoosmotique (ce chiffre de $107 / \mathrm{ml}$ fait l'objet de contestations dans l'auditoire, certains faisant remarquer que le nombre total de spermatozoïdes mobiles dans l'éjaculat serait un paramètre plus intéressant à prendre en compte).

PALERmo (New York) a traité des aneuploïdies, conséquences des techniques de micromanipulation des gamètes humains. Les zygotes obtenus après FIV, "SUZI", ou "ICSI" et qui présentaient un nombre anormal de globules polaires et/ou de pronucléi
(PN), ont ét analysés du point de vue génomique par hybridation in situ. Après FIV et ICSI, les formes présentant un PN unique étaient rares (3\%), les triploïdies par dispermie étaient retrouvées à $5 \%$ et $7 \%$ respectivement (les digynies étaient exceptionnelles). Parmi les formes à $1 \mathrm{PN}$, beaucoup étaient de fait des œufs fécondés et non activés. La situation est apparue très différente en ce qui concerne l'ICSI, où la plupart de ces œufs à 1 PN étaient le résultat d'une activation parthénogénétique. On notait également $4 \%$ de triploïdies, en totalité d'origine digynique. L'auteur a insisté sur le rôle du centriole d'origine spermatique dans la participation au 1er fuseau de division (démontré depuis peu chez l'homme), qui peut aboutir à une régulation non mosaïque dans le cas d'une digynie, mais pas dans celui d'une dispermie (car il y a alors 2 centrioles "paternels", d'où la constitution d'un fuseau tripolaire).

Devroey (Bruxelles) a confirmé les excellents résultats obtenus en collaboration avec l'équipe de Van Steirteghem pour ce qui concerne la technique de micro injection intra-cytoplasmique (ICSI), avec plus de $50 \%$ d'ovocytes fécondés, et plus de $30 \%$ de grossesse évolutive par transfert. Les échecs complets de fécondation sont très rares, même avec des spermes jugés "catastrophiques". Globalement, les auteurs n'ont pas trouvé de corrélation entre le taux de fécondation et les caractéristiques initiales du sperme (seule une nécrospermie élevée est de plus mauvais pronostic); en particulier, une tératozoospermie avoisinant les $100 \%$ ne constitue pas une contre-indication. Sur 274 amniocentèses ou villosités choriales, les auteurs ont observé un seul caryotype anormal (47, XXY). A ce jour, ils faisaient état de 242 enfants nés, dont 5 étaient porteurs de malformations. Ce taux de $2,1 \%$ correspond à celui attendu dans les naissances issues de fécondation naturelle. Même s'il est encore trop tôt pour pouvoir affirmer l'inocuité de cette technique, ces données sont néanmoins rassurantes. 
Silber (St Louis, USA) a cloturé ce symposium en rapportant les résultats de l'ICSI avec des spermatozoïdes ponctionnés dans l'épididyme (en collaboration avec l'équipe bruxelloise). Dans 3 cas, on a utilisé des spermatozoïdes épididymaires qui avaient été congelés lors d'une ponction précédente; dans 16 cas, la ponction épididymaire a été "blanche" et on a dû utiliser des spermatozoïdes testiculaires, récupérés après dilacération d'une biopsie.
Les résultats sont résumés dans le tableau suivant :

Ces résultats se passent de commentaires ! Il faut néanmoins préciser qu'ils ont été obtenus grâce à une collaboration entre 2 équipes considérées actuellement comme les plus performantes dans leur spécialité respective.

Compte-rendu de J.F. GUERIN

\begin{tabular}{lccccc}
\hline $\begin{array}{l}\text { Origine des } \\
\text { spermatozoïdes }\end{array}$ & $\begin{array}{c}\text { N } \\
\text { cycles }\end{array}$ & $\begin{array}{c}\text { Taux de } \\
\text { fécondation }\end{array}$ & $\begin{array}{c}\text { Taux de } \\
\text { clivage }\end{array}$ & $\begin{array}{c}\text { Taux de } \\
\text { transfert } \\
(>1 \text { embryon) }\end{array}$ & $\begin{array}{c}\text { Taux de } \\
\text { grossesse }\end{array}$ \\
\hline $\begin{array}{l}\text { épididymaire } \\
\text { frais }\end{array}$ & 29 & $44 \%$ & $28 \%$ & $93 \%$ & $52 \%$ \\
$\begin{array}{l}\text { épididymaire } \\
\text { congelés }\end{array}$ & 3 & $28 \%$ & $23 \%$ & $100 \%$ & $67 \%$ \\
$\begin{array}{l}\text { biopsie } \\
\text { testiculaire }\end{array}$ & 16 & $44 \%$ & $30 \%$ & $75 \%$ & $31 \%$ \\
\hline \begin{tabular}{l} 
Total \\
\hline
\end{tabular} & $\mathbf{4 8}$ & $\mathbf{4 3 \%}$ & $\mathbf{3 1 \%}$ & $\mathbf{8 3 \%}$ & $\mathbf{4 6 \%}$ \\
\hline
\end{tabular}

